\title{
Transverse broadband impedance reduction techniques in a heavy ion accelerator
}

\author{
X. Q. Chen $\odot,{ }^{1,2,3}$ G. Y. Zhu,${ }^{1,3,{ }^{*}}$ F. Caspers, ${ }^{4}$ J. C. Yang $\odot,{ }^{1,3, \dagger}$ J. X. Wu ${ }^{1,3}$ G. D. Shen,,${ }^{1,3}$ \\ J. Liu $\oplus^{1,3}$ J. W. Xia, ${ }^{1,3}$ Y. Zhang, ${ }^{1,3}$ S. Ruan, ${ }^{1,3}$ G. Wang, ${ }^{1,3}$ L. P. Yao, ${ }^{1,3}$ F. C. Cai $\oplus^{1,2,3}$ \\ H. Ren, ${ }^{1,2,3}$ Q. Y. Kong, ${ }^{1,2,3}$ and Y.Z. Gao ${ }^{1,2,3}$ \\ ${ }^{1}$ Institute of Modern Physics, Chinese Academy of Sciences, Lanzhou 730000, China \\ ${ }^{2}$ University of Chinese Academy of Sciences, Beijing 100049, China \\ ${ }^{3}$ Huizhou Research Center of Ion Science, Huizhou 516003, China \\ ${ }^{4}$ CERN, 1211 Geneva 23, Switzerland
}

(Received 21 November 2019; accepted 21 February 2020; published 11 March 2020)

\begin{abstract}
The transverse broadband impedances of major components in the BRing (booster ring) of HIAF (High Intensity Heavy-ion Accelerator Facility) are estimated using the analytical formulas or the wakefield solver in the CST Studio Suite. At low frequency, the transverse broadband impedance model of BRing is $Z_{1}^{H}(\omega)=-417.14 i \mathrm{k} \Omega / \mathrm{m}$ (horizontal) and $Z_{1}^{V}(\omega)=-530.19 i \mathrm{k} \Omega / \mathrm{m}($ vertical), which are larger than the threshold impedance for the transverse mode-coupling instability. The ceramic rings in the vacuum chamber are the primary source of impedance. With a goal of mitigating the instability by reducing the impedance of ceramic rings, a high conductivity coating is discussed in detail. In addition, a prototype of ceramic rings-loaded thin-wall vacuum chamber is manufactured and the impedance measurements are performed. When ceramic rings are coated by $2 \mu \mathrm{m}$-copper, the CST simulation and experiment results show that the transverse broadband impedance of ceramic rings-loaded thin-wall vacuum chamber can be reduced from $Z_{1}^{H}(\omega)=-291.69 i \mathrm{k} \Omega / \mathrm{m}$ and $Z_{1}^{V}(\omega)=-352.37 i \mathrm{k} \Omega / \mathrm{m}$ to $Z_{1}^{H}(\omega)=-46.16 i \mathrm{k} \Omega / \mathrm{m}$ and $Z_{1}^{V}(\omega)=-64.56 i \mathrm{k} \Omega / \mathrm{m}$. Furthermore, in this case the transverse broadband impedance model of BRing is reduced by more than $50 \%$ to $Z_{1}^{H}(\omega)=-171.61 i \mathrm{k} \Omega / \mathrm{m}$ and $Z_{1}^{V}(\omega)=-242.38 i \mathrm{k} \Omega / \mathrm{m}$.
\end{abstract}

DOI: 10.1103/PhysRevAccelBeams.23.034402

\section{INTRODUCTION}

BRing (booster ring) is the main accelerator of HIAF (High Intensity Heavy-ion Accelerator Facility) project that is proposed and designed by IMP (Institute of Modern Physics, Chinese Academy of Sciences) and is an international advanced heavy ion accelerator facility [1]. The BRing is designed for a maximum momentum rigidity of $34 \mathrm{Tm}$ and a ramping speed of $12 \mathrm{~T} / \mathrm{s}$ with a circumference of $569.1 \mathrm{~m}$, and can accumulate and accelerate the ${ }^{238} \mathrm{U}^{35+}$ beam from $17 \mathrm{MeV} / \mathrm{u}$ to an energy of $835 \mathrm{MeV} / \mathrm{u}$ and the $\mathrm{p}$ beam from $48 \mathrm{MeV} / \mathrm{u}$ to an energy of $9.3 \mathrm{GeV} / \mathrm{u}$. The general layout of BRing complex is shown in Fig. 1 [2].

\footnotetext{
*Corresponding author. zhuguangyu@impcas.ac.cn

Corresponding author. yangjch@impcas.ac.cn
}

Published by the American Physical Society under the terms of the Creative Commons Attribution 4.0 International license. Further distribution of this work must maintain attribution to the author(s) and the published article's title, journal citation, and DOI.

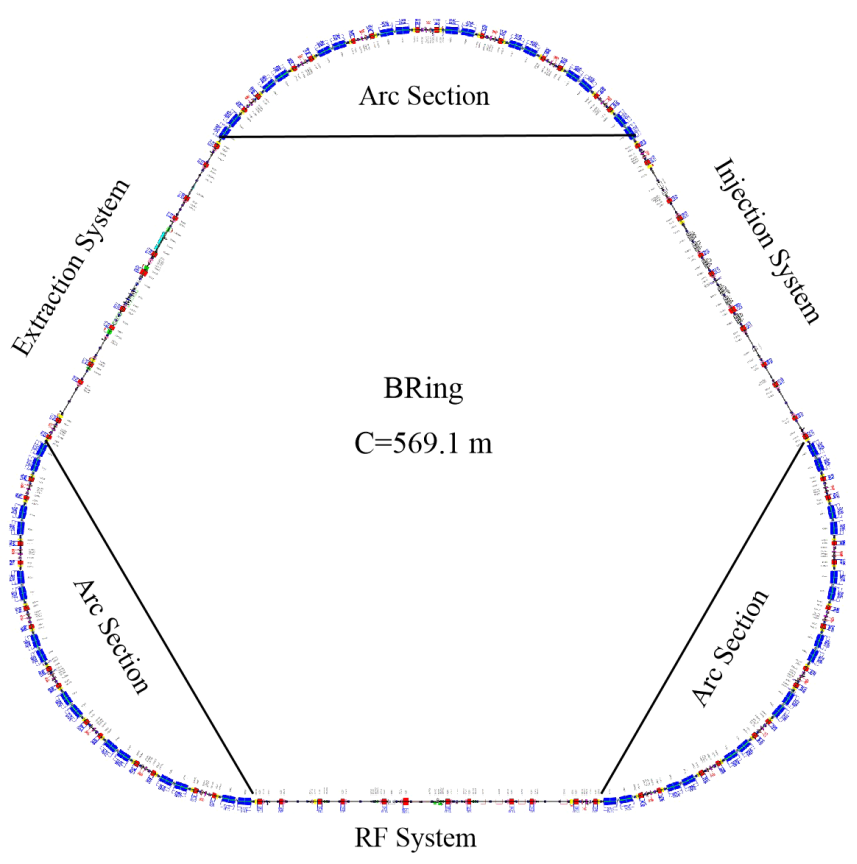

FIG. 1. The general layout of BRing complex. 
The transverse mode-coupling instability stimulated by transverse broadband impedance can have many detrimental impacts on the beam in the high intensity accelerators. The beam may suffer distortion, displacement, or emittance growth, which result in the beam loss $[3,4]$. Therefore, the accurate evaluation of transverse broadband impedance is significant for simulating the transverse mode-coupling instability. Besides, the impedance reduction techniques need to be considered when the beam coupling impedance is larger than the threshold impedance for instability. In the BRing, the transverse broadband impedances mainly come from ceramic rings in the vacuum chamber, bellows, holes, steps, collimators, and beam position monitors. Because the ceramic rings in the vacuum chamber will generate a larger broadband impedance, the evaluation of transverse broadband impedance model and the consideration of impedance reduction techniques are inevitable.

In this paper, some dominant broadband impedance sources in the BRing are considered and estimated using the analytical formulas or the wakefield solver in the CST Studio Suite [5], which leads to a BRing transverse broadband impedance model. In consideration of mitigating the transverse mode-coupling instability, the impedance reduction techniques are considered and the impedance reduction of ceramic rings is discussed in detail. In addition, the impedance measurements are preformed to make sure that the impedance reduction techniques are feasible.

\section{TRANSVERSE BROADBAND IMPEDANCE MODEL IN THE BRING}

In the BRing, the transverse broadband impedances mainly come from ceramic rings in the vacuum chamber, bellows, holes, steps, collimators and beam position monitors, which are estimated using the analytical formulas or the wakefield solver in the CST Studio Suite. The total transverse broadband impedance is the sum of transverse broadband impedance with the local betatron function used as a weight, the analytical formula is [4]

$$
\left.Z_{1}^{\perp}(\omega)\right|_{\text {total }}=\sum \frac{\beta_{\perp} Z_{1}^{\perp}(\omega)}{\bar{\beta}_{\perp}} .
$$

$\beta_{\perp}$ is the local betatron function, $\bar{\beta}_{\perp}$ is the average betatron function. In the BRing, the horizontal average betatron function $\bar{\beta}_{\perp, H}$ is $9.564 \mathrm{~m}$ and the vertical $\bar{\beta}_{\perp, V}$ is $9.605 \mathrm{~m}$.

Besides, the bunch length (root-mean-square) of BRing is larger than $10 \mathrm{~m}$ and the interested frequency of broadband impedance is lower than $300 \mathrm{MHz}$ [6]. Therefore, all the impedance spectrums are given with a wider frequency range from $\mathrm{DC}$ to $500 \mathrm{MHz}$ to obtain a more accurate result.

Furthermore, the theoretical calculations, simulations, and experimental methods are based on the assumption of ultrarelativistic particles in order to facilitate the impedance

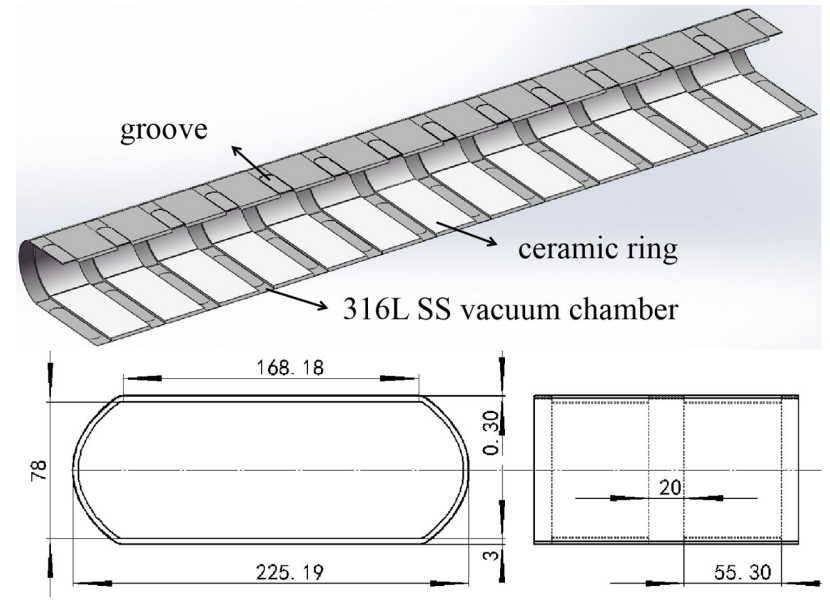

FIG. 2. Ceramic rings-loaded thin-wall 316L SS vacuum chamber.

analysis. Because the beams of BRing are not ultrarelativistic particles, the impedance model of ultrarelativistic particles will be transfer to the impedance model of low beta particles when analyzing the beam collective instabilities in the BRing.

\section{A. Ceramic rings in the vacuum chamber}

In the process of accelerating beam, the fast ramping magnetic field will generate a non-negligible eddy current in the metal vacuum chamber. In order to reduce the attenuation and hysteresis effect of magnetic field caused by eddy-current field, the $0.3 \mathrm{~mm}$-thick ultrathin $316 \mathrm{~L} \mathrm{SS}$ (stainless steel) vacuum chamber is selected in the BRing [7]. At the same time, the ceramic rings are selected to support the ultrathin vacuum chamber [8]. The cross section of the vacuum chamber is shown in Fig. 2. The grooves, which are located on the gap of the ceramic rings, are designed to avoid the mechanical movement of the ceramic rings.

After simplifying a racetracklike ceramic ring into an elliptic ceramic ring with a length of major axis $112.6 \mathrm{~mm}$ and minor axis $39 \mathrm{~mm}$, the transverse impedance of ceramic ring at low frequency is estimated using the classical formula as [9]

$Z_{1}^{\perp}(\omega)=\frac{Z_{0} L}{2 \pi r^{2}}\left[\frac{2 \varepsilon \tan \delta_{\varepsilon}}{(\varepsilon+\alpha)^{2}}-i\left(\frac{\varepsilon-\alpha}{\varepsilon+\alpha}-\frac{r^{2}}{(r+\Delta)^{2}}\right)\right] \cdot G\left(\nu_{0}\right)$

The material of ceramic ring is ZTA (zirconia toughened alumina), $Z_{0}=377 \Omega$ is the characteristic impedance of free space, $\varepsilon=20$ is the relative dielectric constant of ZTA, $\tan \delta_{\varepsilon}$ is the loss-tangent $\left(10^{-4}\right.$ for alumina). $\alpha=\frac{(r+\Delta)^{2}-r^{2}}{(r+\Delta)^{2}+r^{2}}$, $\Delta=3 \mathrm{~mm}$ is the thickness of ceramic ring, $r=39 \mathrm{~mm}$ is the minor axis length of ceramic ring. $L=55.3 \mathrm{~mm}$ is the length of ceramic ring and there are thirteen ceramic rings 


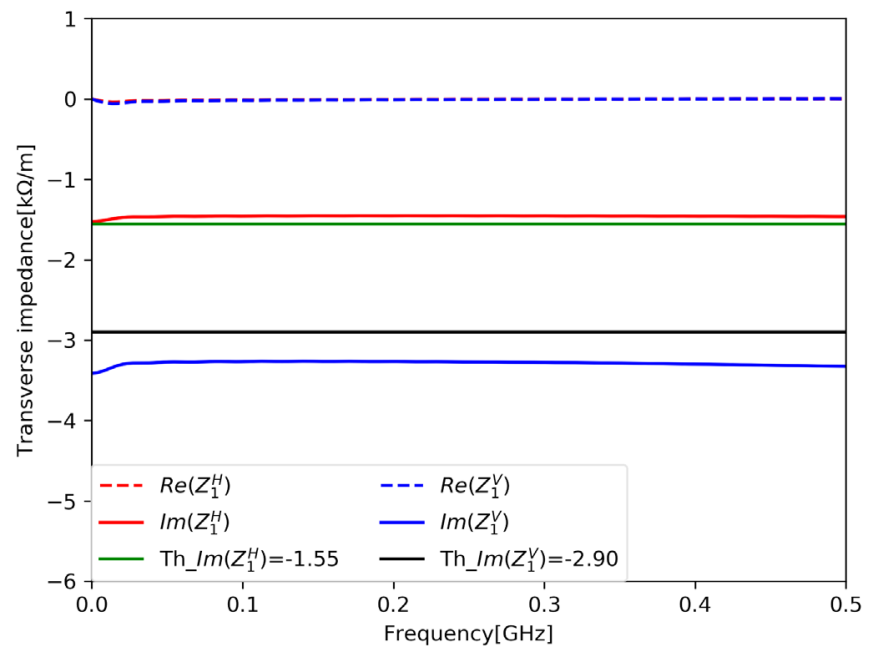

FIG. 3. Real and imaginary part of transverse broadband impedance for the ceramic rings. $\operatorname{Re}\left(Z_{1}^{H}\right)$ and $\operatorname{Im}\left(Z_{1}^{H}\right)$ are the real and imaginary part of horizontal broadband impedance, $\operatorname{Re}\left(Z_{1}^{V}\right)$ and $\operatorname{Im}\left(Z_{1}^{V}\right)$ are the real and imaginary part of vertical broadband impedance, $T h \_\operatorname{Im}\left(Z_{1}^{H}\right)$ and $T h \_\operatorname{Im}\left(Z_{1}^{V}\right)$ are the theoretical value of the imaginary part of horizontal and vertical broadband impedance.

in the one-meter vacuum chamber of BRing. $G\left(\nu_{0}\right)$ is the form factor of ellipse [10], the horizontal form factor $G_{1 x}\left(\nu_{0}\right)=0.45$ and the vertical form factor $G_{1 y}\left(\nu_{0}\right)=$ 0.84 for the ellipse with a length of major axis $112.6 \mathrm{~mm}$ and minor axis $39 \mathrm{~mm}$. For the ceramic rings, because the loss-tangent $\left(10^{-4}\right.$ for alumina $)$ is very small, the real part of transverse impedance can be neglected.

For the one-meter ceramic rings-loaded thin-wall vacuum chamber, transverse broadband impedance is estimated using Eq. (2) and simulated using the wakefield solver in the CST Studio Suite, and the results are shown in Fig. 3. At low frequency (below $0.3 \mathrm{GHz}$ ), the theoretical value of horizontal broadband impedance is $-1554.59 i \Omega / \mathrm{m}$ and the CST simulation result is approximately $-1545 i \Omega / \mathrm{m}$, the theoretical value of vertical broadband impedance is $-2901.89 i \Omega / \mathrm{m}$ and the CST simulation result is approximately $-3264 i \Omega / \mathrm{m}$. The divergence between theoretical value and CST simulation result mainly come from the elliptic cross section simplified. The total transverse broadband impedance of ceramic rings is estimated using the Eq. (1) with CST simulation results, which gives the horizontal broadband impedance $Z_{1}^{H}(\omega)=$ $-291.69 i \mathrm{k} \Omega / \mathrm{m}$ and the vertical broadband impedance $Z_{1}^{V}(\omega)=-352.37 i \mathrm{k} \Omega / \mathrm{m}$.

\section{B. Bellows}

There are 150 bellows in the BRing, which are mainly located on the vacuum chamber end at magnets and beam diagnostics instruments position. Bellows will break the smooth wall of vacuum chamber and introduce a

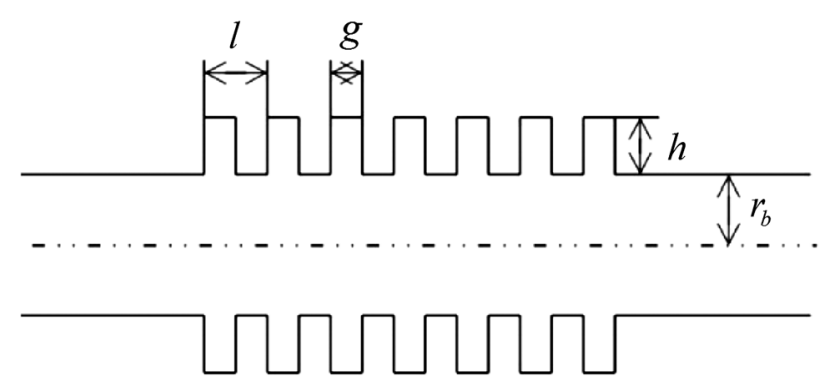

FIG. 4. A schematic view of bellows.

broadband impedance. A schematic view of the bellows is shown in Fig. 4.

The transverse broadband impedance of the bellows is estimated using the classical formula as [11]

$$
Z_{1}^{\perp}(\omega)=-i \frac{Z_{0} g h L_{b}}{\pi l r_{b}^{3}}
$$

$L_{b}=127 \mathrm{~mm}$ is the length of bellows, $r_{b}=100 \mathrm{~mm}$ is the radius of the bellows. For the period structure of the bellows, $l=12 \mathrm{~mm}$ is the cell length, $g=6 \mathrm{~mm}$ is the gap, and $h=19.36 \mathrm{~mm}$ is the height. The total transverse broadband impedance of the bellows is estimated using Eq. (3) and Eq. (1), which gives the horizontal broadband impedance $Z_{1}^{H}(\omega)=-35.56 i \mathrm{k} \Omega / \mathrm{m}$ and the vertical broadband impedance $Z_{1}^{V}(\omega)=-45.26 i \mathrm{k} \Omega / \mathrm{m}$.

\section{Collimators}

There are 27 collimator systems in the BRing, and each collimator has 2 collimating blocks with $100 \mathrm{~mm}$-length, $40 \mathrm{~mm}$-width and $40 \mathrm{~mm}$-height and 7 holes with $75 \mathrm{~mm}$ radius on the vacuum chamber. The transverse broadband impedance of collimator is simulated using the wakefield solver in the CST Studio Suite. Half of the 3D CST-model of collimator is shown in the Fig. 5 and the simulation results are shown in Fig. 6.

At low frequency (below $0.3 \mathrm{GHz}$ ), the CST simulation result of horizontal broadband impedance for one

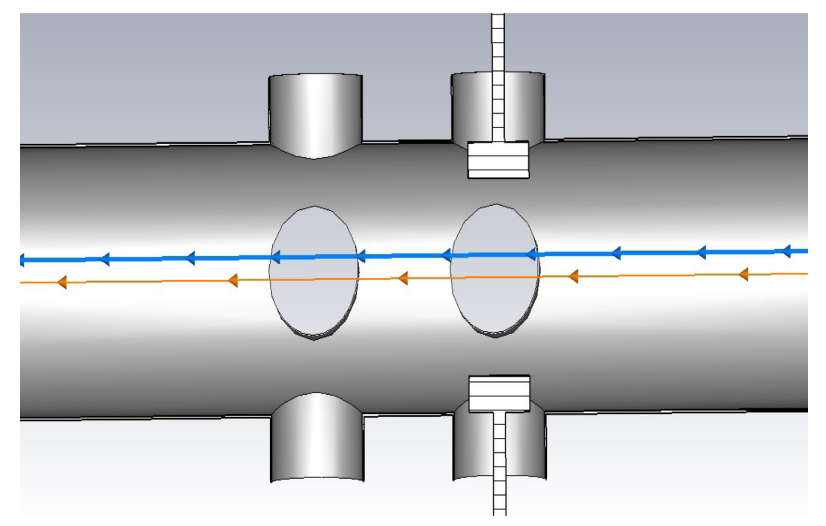

FIG. 5. Half of the 3D CST-model of collimator. 


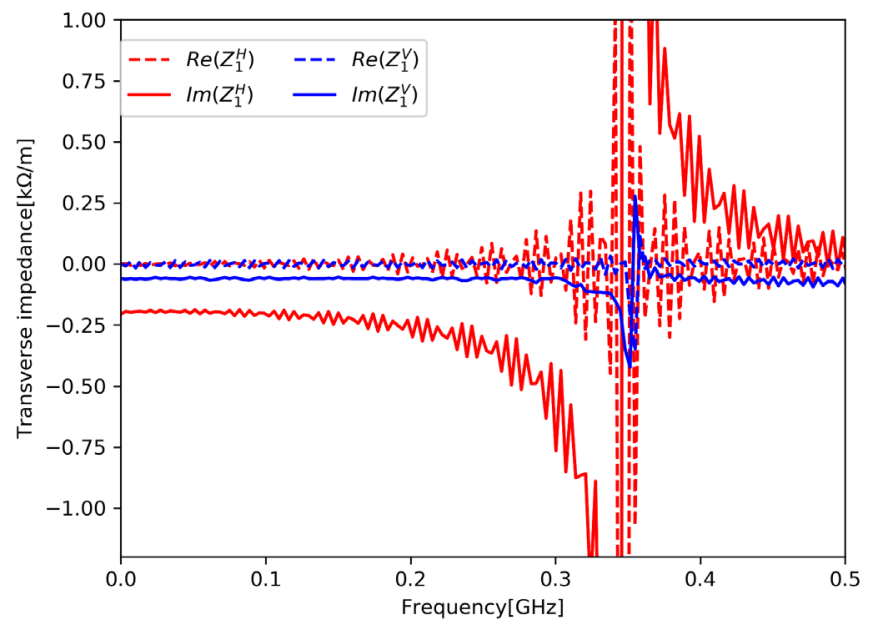

FIG. 6. Real and imaginary part of transverse broadband impedance for the collimator simulated using CST.

collimator system is approximately $-200 i \Omega / \mathrm{m}$ and the vertical broadband impedance is approximately $-75 i \Omega / \mathrm{m}$. The total transverse broadband impedance of collimators is estimated using the Eq. (1) with CST simulation results, which gives the horizontal broadband impedance $Z_{1}^{H}(\omega)=-3.53 i \mathrm{k} \Omega / \mathrm{m}$ and the vertical broadband impedance $Z_{1}^{V}(\omega)=-4.24 i \mathrm{k} \Omega / \mathrm{m}$.

\section{Holes}

In the BRing, the holes on the vacuum chamber are mainly introduced by the vacuum pumps whose port radii are $100 \mathrm{~mm}$ or $75 \mathrm{~mm}$. The $75 \mathrm{~mm}$-radius pumps are 50 NEG (nonevaporable getter) pumps, 190 SIPs (sputter ion pump) and 25 TMPs (turbo molecular pump), and the $100 \mathrm{~mm}$-radius pumps are 142 TSPs (titanium sublimation pump). The transverse broadband impedance of hole is estimated using the classical formula as [12]

$$
Z_{1}^{\perp}(\omega)=-i \frac{Z_{0} r_{h}^{3}}{3 \pi^{2} r_{v}^{4}}
$$

$r_{v}=100 \mathrm{~mm}$ is the radius of vacuum chamber at vacuum pumps position, $r_{h}$ is the port radius of vacuum pumps. The total transverse broadband impedance of holes is estimated using Eq. (4) and Eq. (1), which gives the horizontal broadband impedance $Z_{1}^{H}(\omega)=-32.13 i \mathrm{k} \Omega / \mathrm{m}$ and the vertical broadband impedance $Z_{1}^{V}(\omega)=-53.52 i \mathrm{k} \Omega / \mathrm{m}$.

\section{E. Flanges and transition steps}

It is necessary to design the varying width of beam pipe for the use of beam instrumentation, insertion devices, flanges, etc., in a synchrotron. In the BRing, the variations of radius for beam pipe are shown in Fig. 7.

After considering the presence of changes in the beam pipe diameters, the transverse broadband impedance of

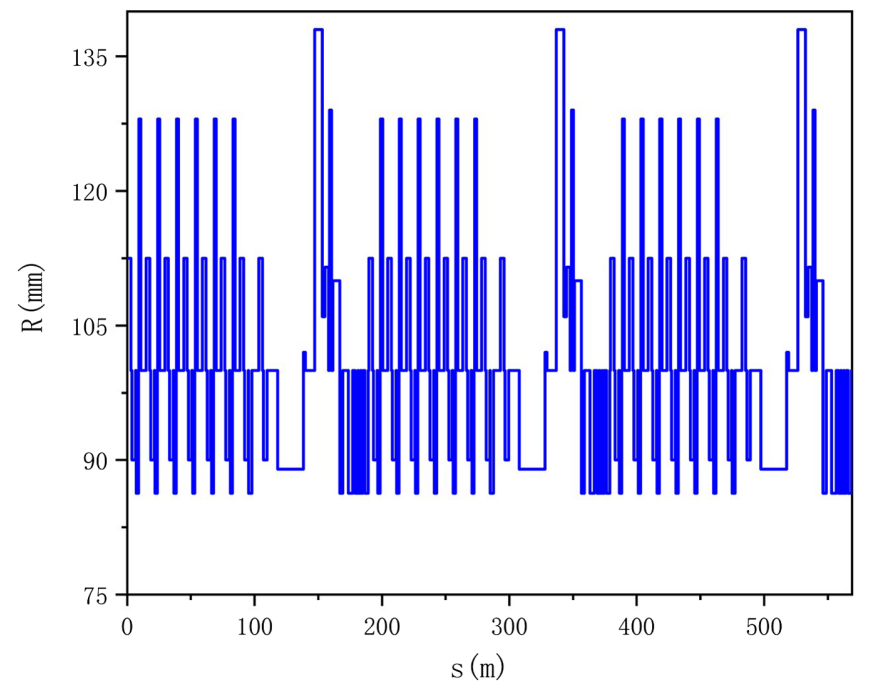

(a)

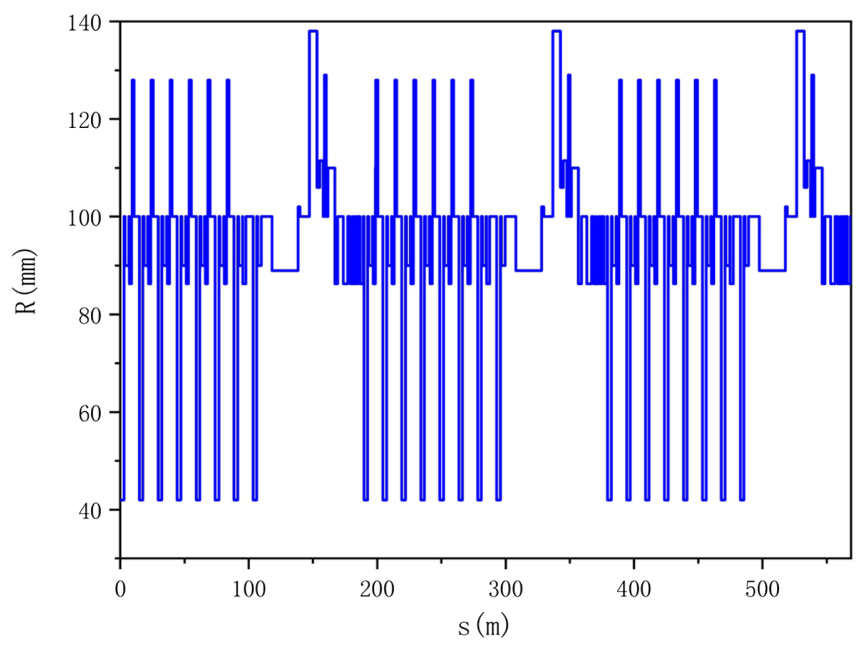

(b)

FIG. 7. Variations of radius for beam pipe. (a) Horizontal (b) Vertical.

flanges and transition steps are estimated by the classical formula as [13]

$$
Z_{1}^{\perp}(\omega)=-i \frac{Z_{0} r_{c}^{2}}{\pi^{2} r_{t}^{3}}\left(\ln \frac{2 \pi r_{t}}{r_{c}}+0.5\right) .
$$

$r_{t}=100 \mathrm{~mm}$ is the initial radius of beam pipe, $r_{c}$ is the height of object and is similar to the height of period structure of the bellows. The total transverse broadband impedance of flanges and transition steps are estimated using Eq. (5) and Eq. (1), which give the horizontal broadband impedance $Z_{1}^{H}(\omega)=-41.51 i \mathrm{k} \Omega / \mathrm{m}$ and the vertical broadband impedance $Z_{1}^{V}(\omega)=-41.45 i \mathrm{k} \Omega / \mathrm{m}$.

\section{F. Beam position monitors}

There are 39 linear-cut BPMs (beam position monitors) in the BRing, and each BPM has four $0.3 \mathrm{~mm}$-thick round 


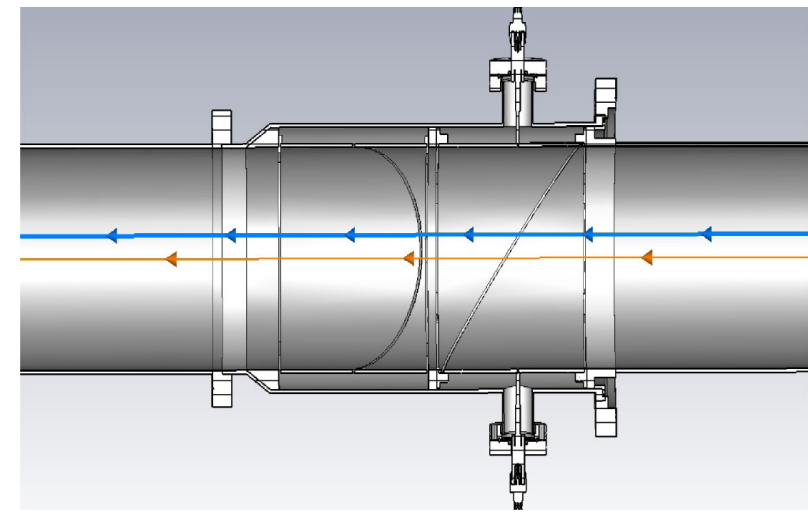

FIG. 8. Half of the 3D CST-model of BPM.

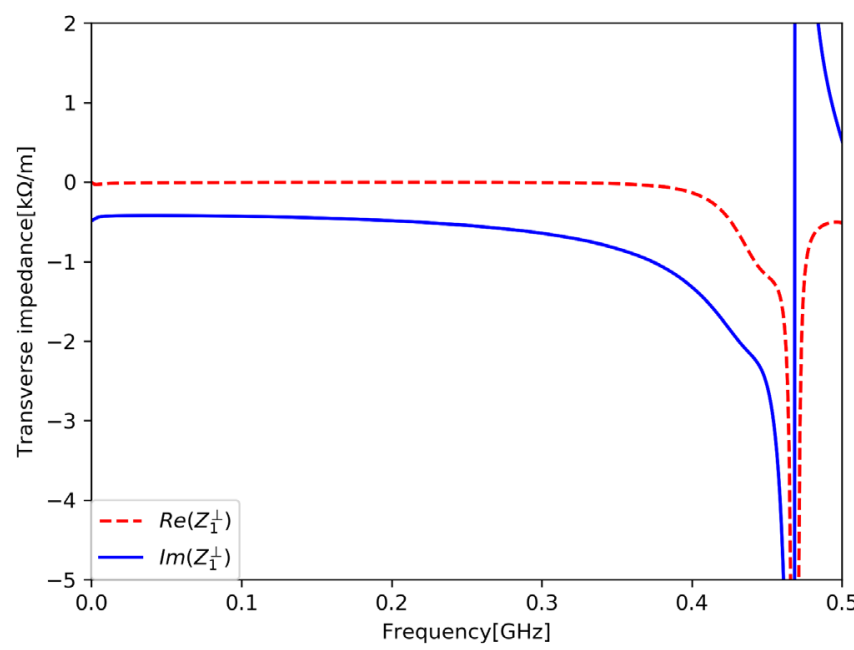

FIG. 9. Real and imaginary part of transverse broadband impedance for the BPM simulated using CST.

electrode plates with a $95 \mathrm{~mm}$-radius. With a goal of reducing the coupling between the electrode plates, the gap of electrode plates is optimized and the grounded ceramic ring is used to insulate the electrode plates and beam pipe. Because of the complicated structure of BPM, the transverse broadband impedance cannot be calculated using the analytical method, and the wakefield solver in the CST Studio Suite has been used to simulate it. Half of the 3D CST-model of BPM is shown in Fig. 8 and the simulation result is shown in Fig. 9.

At low frequency (below $0.3 \mathrm{GHz}$ ), the CST simulation result of transverse broadband impedance for one BPM is approximately $-450 i \Omega / \mathrm{m}$. The total transverse broadband impedance of BPMs is estimated using Eq. (1) with CST simulation result, which gives the horizontal broadband impedance $Z_{1}^{H}(\omega)=-13.18 i \mathrm{k} \Omega / \mathrm{m}$ and the vertical broadband impedance $Z_{1}^{V}(\omega)=-41.80 i \mathrm{k} \Omega / \mathrm{m}$.

In fact, the transverse broadband impedance model of BRing can be approximated to the RLC model of low-Q cavity and given by

$$
Z_{1}^{\perp}(\omega)=\frac{R_{s} \omega_{r}}{\omega+i Q\left(\omega_{r}-\frac{\omega^{2}}{\omega_{r}}\right)} .
$$

$R_{s}$ is the shunt impedance, $Q$ is the quality factor and $\omega_{r}$ is the resonant frequency. For the transverse broadband impedance model of BRing, $R_{s}$ is the absolute value of impedances which are given by the above calculation results and shown in the Table I. $Q=1 . \omega_{r}=2 \pi f_{r}$ is given by the cutoff frequency $\left(f_{r}=0.38 \mathrm{c} / \mathrm{b}\right)$ of beam pipe, $c=3 \times 10^{8} \mathrm{~m} / \mathrm{s}$ is the speed of light, $b=0.1 \mathrm{~m}$ is the average radius of beam pipe. The transverse broadband impedance model of BRing is given by

$$
\begin{aligned}
& Z_{1}^{H}(\omega)=\frac{4.17 \times 10^{5}}{\frac{\omega}{7.16 \times 10^{9}}+i\left(1-\frac{\omega^{2}}{5.13 \times 10^{19}}\right)}(\Omega / \mathrm{m}), \\
& Z_{1}^{V}(\omega)=\frac{5.30 \times 10^{5}}{\frac{\omega}{7.16 \times 10^{9}}+i\left(1-\frac{\omega^{2}}{5.13 \times 10^{19}}\right)}(\Omega / \mathrm{m}) .
\end{aligned}
$$

In the BRing, the total transverse broadband impedances are larger than the threshold impedance for the transverse mode-coupling instability [6]. The chromaticity, octupole magnets and feed-back system are often considered to mitigate the instability, but a large chromaticity will cause a large shift of incoherent tune, a large strength octupole magnet will cause the reduction of dynamic aperture and the design of feedback system is difficult. Therefore, the impedance reduction techniques are the first consideration.

\section{IMPEDANCE REDUCTION TECHNIQUES}

There are a number of ways in which it is possible to reduce the beam coupling impedance. The tapering of step transition is often used to reduce the impedance of flanges and transition steps. The transition pieces are often used to reduce the impedance of bellows, holes and so on. A high conductivity coating is often used to reduce the impedance of collimators, beam instrumentation and numerous other devices $[14,15]$. According to the transverse broadband impedance for the components of BRing in the Table I, the

TABLE I. Transverse broadband impedances for the components of BRing.

\begin{tabular}{lccccccc}
\hline \hline Components & Ceramic rings & Bellows & Holes & Flanges and transition steps & BPMs & Collimators & Total \\
\hline$Z_{1}^{H}(\omega)[\mathrm{k} \Omega / \mathrm{m}]$ & $-291.69 i$ & $-35.56 i$ & $-32.13 i$ & $-41.54 i$ & $-13.18 i$ & $-3.04 i$ & $-417.14 i$ \\
$Z_{1}^{V}(\omega)[\mathrm{k} \Omega / \mathrm{m}]$ & $-352.37 i$ & $-37.46 i$ & $-53.52 i$ & $-41.45 i$ & $-41.80 i$ & $-3.59 i$ & $-530.19 i$ \\
\hline \hline
\end{tabular}




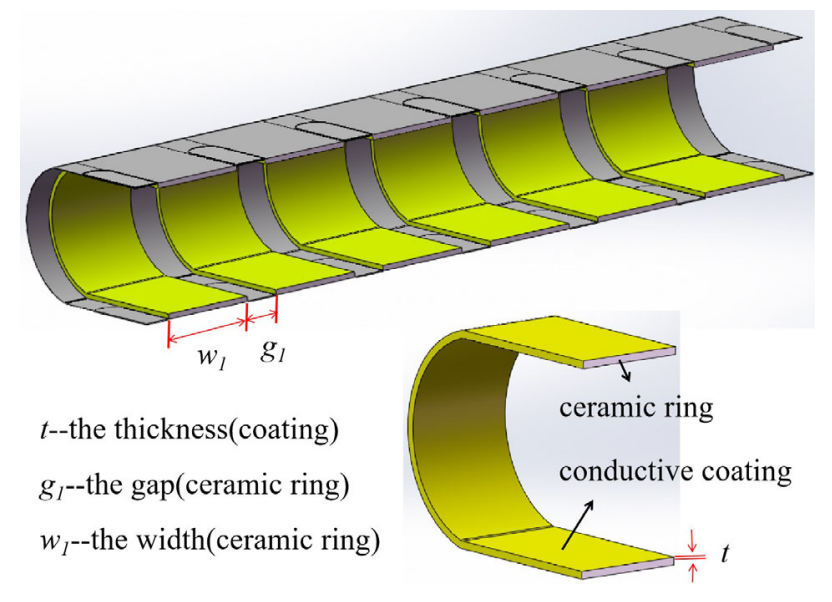

FIG. 10. Ceramic rings with a high conductivity coating.

ceramic rings in the vacuum chamber are the primary source. Besides, the Fig. 6 shows that there is a strong resonance around $0.35 \mathrm{GHz}$ for the transverse impedance of collimators and the lossy ferrite rings inside the stem will be considered to dampen the resonance. Figure 9 shows that there is a strong resonance around $0.45 \mathrm{GHz}$ for the transverse impedance of BPM and the gap of electrode plates and the grounded ceramic ring will be further optimized to move the resonance point of impedance to the high frequency band (above $0.5 \mathrm{GHz}$ ).

In this paper, the impedance reduction of ceramic rings in the vacuum chamber is our focus. A high conductivity coating is discussed in detail to reduce the transverse broadband impedance of ceramic rings.

\section{A. Conductive coating}

It has been shown that a thin layer of high conductivity material plated on the surface of a poorly conductive material can effectively screen the beam from interacting with the poorly conductive material for a large frequency range $[14,16]$. In the BRing, the ceramic ring made of ZTA is a poorly conducting material and will generates a larger broadband impedance. Therefore, the ceramic ring coated by a high conductivity material is considered to reduce its broadband impedance and the schematic view is shown in Fig. 10. $t$ is the thickness of conductive coating.

The thickness of conductive coating depend on the skin depth of coating material. The skin depth of conductive material is

$$
\delta(\omega)=\sqrt{\frac{2}{\mu_{0} \mu_{r}(\omega) \sigma \omega}} .
$$

$\mu_{0}=4 \pi \times 10^{-7} \mathrm{H} / \mathrm{m}$ is the permeability of vacuum, $\mu_{r}(\omega)$ is the relative permeability of material, $\sigma$ is the conductivity of material and $\omega=2 \pi f$ is the radian frequency. The skin depth of copper $\left(\sigma=5.97 \times 10^{7} \mathrm{~S} / \mathrm{m}\right)$ at $0.3 \mathrm{GHz}$ is

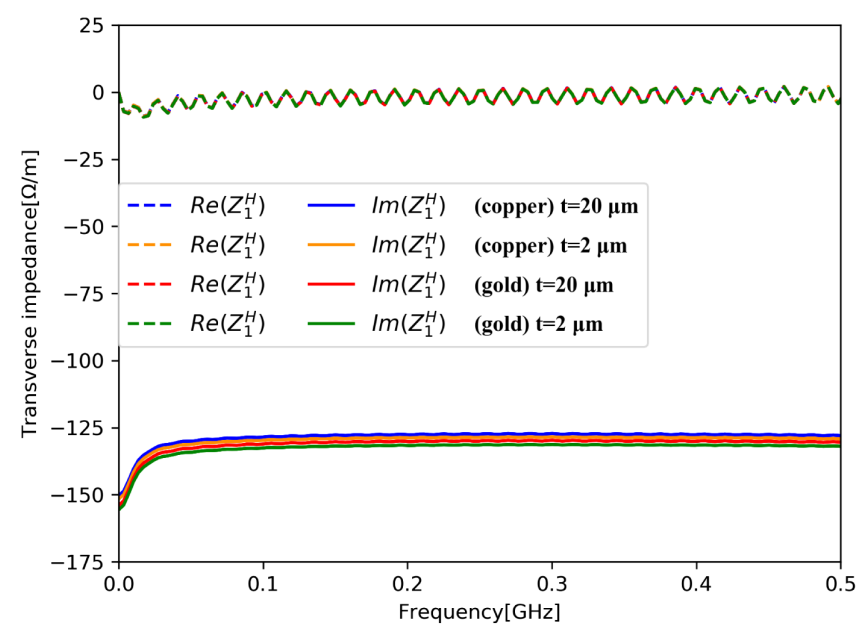

(a)

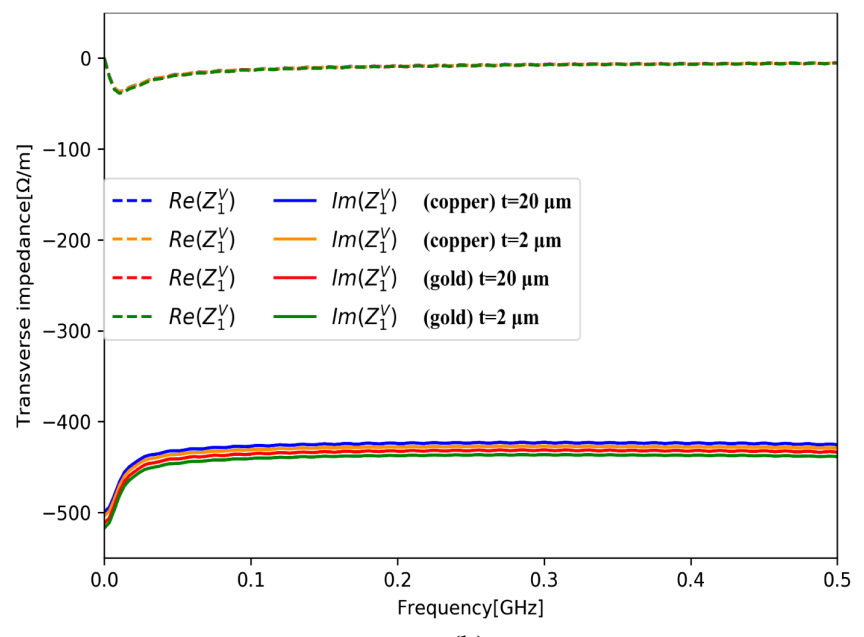

(b)

FIG. 11. Real and imaginary part of transverse broadband impedance for the ceramic rings shield by a conductive coating. (a) Horizontal (b) Vertical.

$\delta(0.3 \mathrm{GHz})=3.76 \mu \mathrm{m}$ and the skin depth of gold $(\sigma=$ $\left.4.56 \times 10^{7} \mathrm{~S} / \mathrm{m}\right)$ at $0.3 \mathrm{GHz}$ is $\delta(0.3 \mathrm{GHz})=4.30 \mu \mathrm{m}$. Therefore, the micron-scale coatings should be considered.

For the one-meter ceramic rings-loaded thin-wall vacuum chamber as shown in Fig. 2, the different thickness of copper and gold coating are used to shield the ceramic rings and transverse broadband impedances are simulated using the wakefield solver in the CST Studio Suite. For the following CST Studio Suite simulations, the local mesh properties are chosen to refine mesh of ultrathin coating of ceramic rings, and the $35 \mathrm{~m}$ wake potential left by a $20 \mathrm{~cm} \mathrm{rms}$ bunch in the model made of $10^{9}$ mesh cells is calculated using the indirect test beam. The simulation results of transverse broadband impedance are shown in Fig. 11.

According to the CST simulation results of Fig. 11, at low frequency (below $0.3 \mathrm{GHz}$ ), the transverse broadband impedance can be reduced more than 7 times when the 


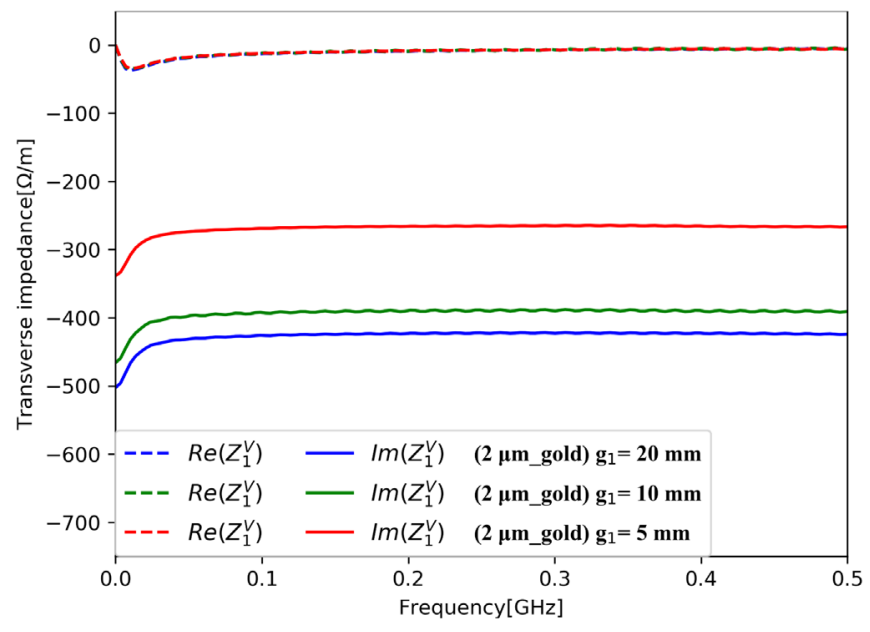

FIG. 12. Real and imaginary part of vertical broadband impedance for the ceramic rings coated by $2 \mu \mathrm{m}$-gold with different gaps.

ceramic rings are coated by $2 \mu \mathrm{m}$-copper. The horizontal broadband impedance is reduced from $Z_{1}^{H}(\omega)=-1545 i \Omega / \mathrm{m}$ to $Z_{1}^{H}(\omega)=-130 i \Omega / \mathrm{m}$ and the vertical broadband impedance is reduced from $Z_{1}^{V}(\omega)=-3264 i \Omega / \mathrm{m}$ to $Z_{1}^{V}(\omega)=-432 i \Omega / \mathrm{m}$. Besides, the impedance reduced by $2 \mu \mathrm{m}$-copper coating is similar to the $20 \mu \mathrm{m}$-copper coating, $2 \mu \mathrm{m}$-gold coating, and $20 \mu \mathrm{m}$-gold coating. The gold coating will be the first consideration because the gas desorption yield and oxidizability of gold is lower than copper.

The ceramic rings coated by $2 \mu \mathrm{m}$-gold coating are taken as an example. The Fig. 2 and the Fig. 10 show that the ceramic rings with $2 \mu \mathrm{m}$-gold coating are more like the bellows, where $w_{1}=55.3 \mathrm{~mm}$ is the width of ceramic rings and $g_{1}=20 \mathrm{~mm}$ is the gap between two ceramic rings. For the one-meter ceramic rings-loaded thin-wall vacuum chamber, the Fig. 12 shows the CST wakefield simulation results of vertical broadband impedances for the ceramic rings coated by $2 \mu \mathrm{m}$-gold with different gap. At low frequency (below $0.3 \mathrm{GHz}$ ), the impedance can be reduced significantly when the gap $g_{1}$ is decreased. But for the same length of vacuum chamber, the number of ceramic ring and the cost of vacuum chamber are increased when the gap $g_{1}$ is decreased. Therefore, it is not necessary to decrease the gap between two ceramic rings.

There are still two parts to note, one is the resonances of impedance spectrum which may be caused by the bad rf contact between the ceramic rings with a high conductivity coating and the $0.3 \mathrm{~mm}$-thick chamber-wall, and the other is the eddy-current effect of conductive coating. From the resonances of impedance spectrum viewpoint, the ceramic ring with $\mathrm{rf}$ shoulders is proposed to achieve the good $\mathrm{rf}$ contact between the ceramic rings with a high conductivity coating and the $0.3 \mathrm{~mm}$-thick chamber-wall. The rf shoulders are located on the two ends of planar portion

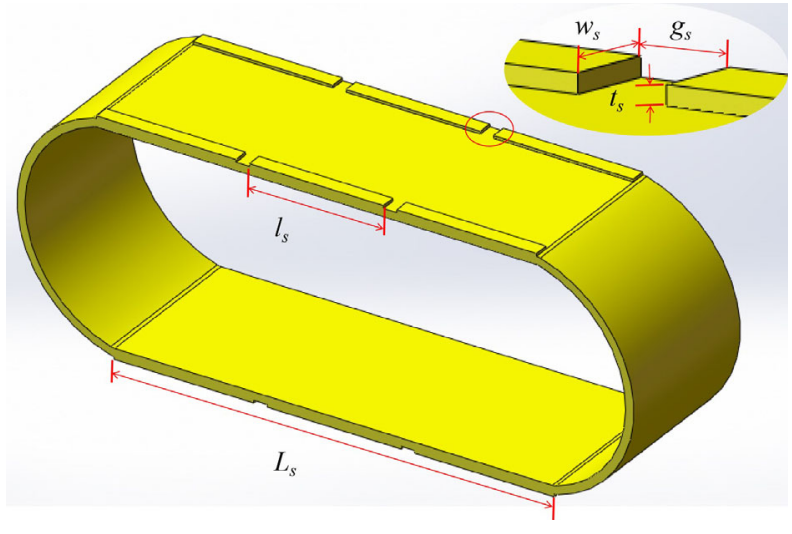

FIG. 13. Ceramic ring with rf shoulders.

of outer surface for each ceramic ring, and the schematic view is shown in Fig. 13. Each rf shoulder is divided into three cells with the same length, $L_{s}=168.18 \mathrm{~mm}$ is the total length, $l_{s}=52.73 \mathrm{~mm}$ is the length of single cell, $w_{s}=5 \mathrm{~mm}$ is the width, $t_{s}=0.1 \mathrm{~mm}$ is the thickness, and $g_{s}=5 \mathrm{~mm}$ is the gap of cells. The gap of cells is designed to make the vacuum section convenient.

From the eddy current viewpoint, the gold conductivity $\left(4.56 \times 10^{7} \mathrm{~S} / \mathrm{m}\right)$ is approximately 32.34 times higher than that of $316 \mathrm{~L}$ stainless steel $\left(1.41 \times 10^{7} \mathrm{~S} / \mathrm{m}\right)$. The $20 \mu \mathrm{m}$-gold coating is not allowed because it is equivalent to adding $0.65 \mathrm{~mm}$-thick $316 \mathrm{~L} \mathrm{SS}$ to the $0.3 \mathrm{~mm}$-thick chamber-wall. The $2 \mu \mathrm{m}$-gold coating is acceptable because it just increases the external magnetic field screening by $21.67 \%$ comparison with the $0.3 \mathrm{~mm}$-thick chamberwall. Furthermore, the ceramic rings coated by a thinner gold coating, for example $0.5 \mu \mathrm{m}$-gold coating, will be better if the impedance reduction of the $0.5 \mu \mathrm{m}$-gold coating is similar to the $2 \mu \mathrm{m}$-gold coating.

In the BRing, a high conductivity coating has been discussed in detail to reduce the transverse broadband impedance of ceramic rings and the impedances have been simulated using the wakefield solver in the CST Studio Suite. In consideration of the mitigation for the eddycurrent effect of conductive coating, the ceramic rings coated by $2 \mu \mathrm{m}$-gold with $20 \mathrm{~mm}$-gap will be considered provisionally to reduce transverse broadband impedance, and the transverse broadband impedance of ceramic rings can be reduced more than 7 times at low frequency (below $0.3 \mathrm{GHz}$ ). Besides, in consideration of the mitigation for the possible resonances of impedance spectrum, the ceramic rings with rf shoulder will be considered.

\section{LABORATORY MEASUREMENT}

In order to make sure the impedance reduction technique for a high conductivity coating is feasible, a prototype of vacuum chamber with ceramic rings ( $2 \mu \mathrm{m}$-copper coating and $20 \mathrm{~mm}$-gap) is manufactured and the impedance measurements are performed. The transverse impedance 


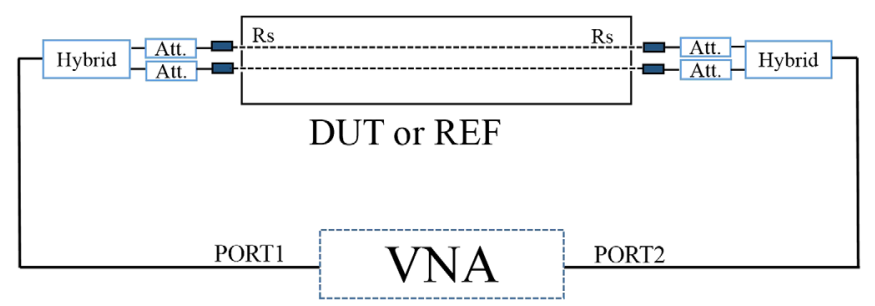

FIG. 14. Schematic view of transverse impedance measurement.

measurement consists of two copper wires driven with opposite phases, and a dipolar field is excited in the DUT (Device Under Test), which only interacts with the fringe field [17]. A schematic view of the impedance measurement is shown in Fig. 14 and the impedance measurement platform is shown in Fig. 15.

A matching resistor $\left(R_{s}\right)$ of $175 \Omega$ per wire is used when the distance between the wires is $10 \mathrm{~mm}$. The different-mode signal is offered by the hybrid and its isolation is bigger than $30 \mathrm{~dB}$. Four $6 \mathrm{~dB}$ attenuators (Att) between the hybrid and the DUT or REF (reference) are involved in the refection from the port. The frequency shift and the electrical length difference can be found by comprising the forward scattering coefficient of DUT and REF. The longitudinal impedance, which is corresponding to the electrical length difference, can be read in the VNA(vector network analyzer), and the transverse impedance can be derived by $P-W$ theory [18]

$$
Z_{1}^{\perp}(\omega)=-\frac{c Z_{0}^{/ /}(\omega)}{\omega D^{2}} .
$$

$D=10 \mathrm{~mm}$ is the spacing of copper wires and $c$ is the speed of light. The comparison between CST simulation and measurement results of one-meter ceramic rings-loaded thin-wall vacuum chamber are shown in Fig. 16.

According to the CST simulation and measurement results of Fig. 16, for the ceramic rings without coating, the CST simulation results of transverse broadband impedance are $Z_{1}^{H}(\omega)=-1545 i \Omega / \mathrm{m}$ and $Z_{1}^{V}(\omega)=$ $-3264 i \Omega / \mathrm{m}$, and the measurement results are $Z_{1}^{H}(\omega)=$ $-1937 i \Omega / \mathrm{m}$ and $Z_{1}^{V}(\omega)=-3879 i \Omega / \mathrm{m}$. When the

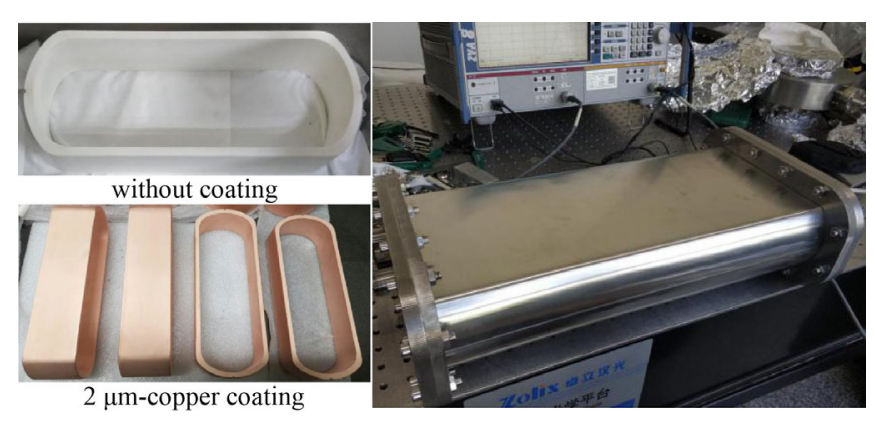

FIG. 15. Impedance measurement platform.

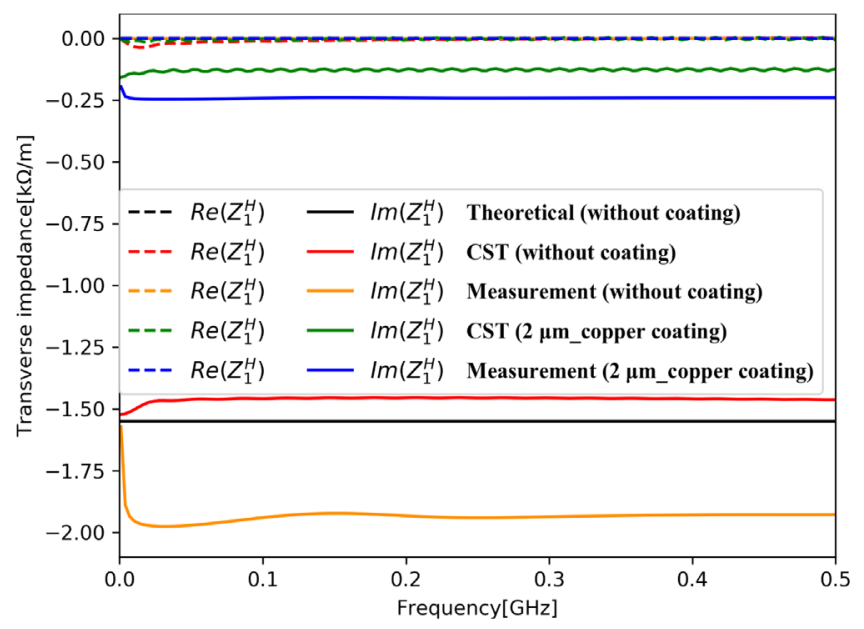

(a)

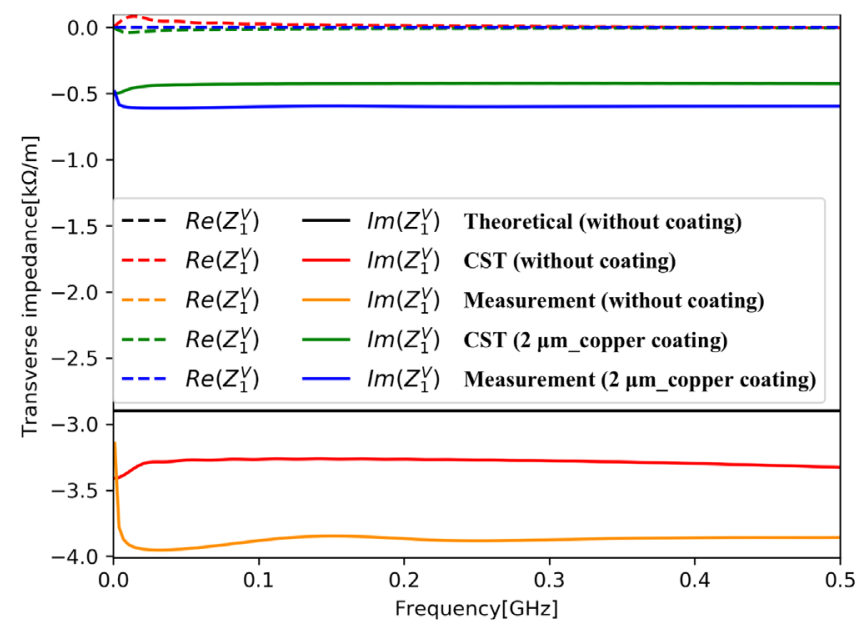

(b)

FIG. 16. CST simulation and measurement results for the transverse broadband impedance of ceramic rings. (a) Horizontal (b) Vertical.

ceramic rings are coated by $2 \mu \mathrm{m}$-copper, the CST simulation results of transverse broadband impedance are $Z_{1}^{H}(\omega)=-130 i \Omega / \mathrm{m}$ and $Z_{1}^{V}(\omega)=-423 i \Omega / \mathrm{m}$, and the measurement results are $Z_{1}^{H}(\omega)=-246 i \Omega / \mathrm{m}$ and $Z_{1}^{V}(\omega)=-598 i \Omega / \mathrm{m}$. The measurement results are larger than the CST simulation results because of measurement inaccuracy and uneven coating.

\section{SUMMARY}

In the BRing, the transverse broadband impedances mainly come from ceramic rings in the vacuum chamber, bellows, holes, steps, collimators, and beam position monitors. They are estimated using the analytical formulas or the wakefield solver in the CST Studio Suite. The transverse broadband impedance model of BRing is given by the Eq. (7), which are larger than the threshold impedance for the transverse mode-coupling instability. 
Besides, the ceramic rings in the vacuum chamber are the primary source of impedance.

Therefore, a high conductivity coating has been discussed in detail to reduce the transverse broadband impedance of ceramic rings. Transverse broadband impedance can be reduced more than 7 times at low frequency (below $0.3 \mathrm{GHz}$ ) when the ceramic rings are coated by $2 \mu \mathrm{m}$-copper or $2 \mu \mathrm{m}$-gold. In order to make sure the impedance reduction technique for a high conductivity coating is feasible, a prototype of vacuum chamber with ceramic rings ( $2 \mu \mathrm{m}$-copper coating and $20 \mathrm{~mm}$-gap) is manufactured and the impedance measurements are performed. According to the CST simulation and measurement results of the one-meter ceramic rings-loaded thin-wall vacuum chamber, when the ceramic rings are coated by $2 \mu \mathrm{m}$-copper, the horizontal broadband impedance is reduced from $Z_{1}^{H}(\omega)=-1545 i \Omega / \mathrm{m}$ to $Z_{1}^{H}(\omega)=$ $-246 i \Omega / \mathrm{m}$ and the vertical broadband impedance is reduced from $Z_{1}^{V}(\omega)=-3264 i \Omega / \mathrm{m}$ to $Z_{1}^{V}(\omega)=$ $-598 i \Omega / \mathrm{m}$. Therefore, the transverse broadband impedance of ceramic rings in the BRing is reduced from $Z_{1}^{H}(\omega)=-291.69 i \mathrm{k} \Omega / \mathrm{m}$ and $Z_{1}^{V}(\omega)=-352.37 i \mathrm{k} \Omega / \mathrm{m}$ to $Z_{1}^{H}(\omega)=-46.16 i \mathrm{k} \Omega / \mathrm{m}$ and $Z_{1}^{V}(\omega)=-64.56 i \mathrm{k} \Omega / \mathrm{m}$, and the transverse broadband impedance model is reduced by more than $50 \%$ to $Z_{1}^{H}(\omega)=-171.61 i \mathrm{k} \Omega / \mathrm{m}$ and $Z_{1}^{V}(\omega)=-242.38 i \mathrm{k} \Omega / \mathrm{m}$. Because the gas desorption yield and oxidizability of gold is lower than copper, the ceramic rings coated by $2 \mu \mathrm{m}$-gold are a better choice and have been considered.

Furthermore, in consideration of the mitigation for the possible resonances of impedance spectrum, the ceramic rings with rf shoulder will be considered. In consideration of the mitigation for the eddy-current effect of conductive coating, the ceramic rings coated by a thinner gold coating, for example $0.5 \mu \mathrm{m}$-gold coating, will be better if the impedance reduction of the $0.5 \mu \mathrm{m}$-gold coating is similar to the $2 \mu \mathrm{m}$-gold coating. But it is difficult to simulate the impedance of ceramic rings coated by $0.5 \mu \mathrm{m}$-gold because the wakefield solver in the CST Studio Suite cannot create a fine mesh in $0.5 \mu \mathrm{m}$-gold coating.

In the next step, in order to make sure that the resonances of impedance spectrum do not appear in a wide frequency range and the impedance reduction of the ceramic rings coated by $0.5 \mu \mathrm{m}$-gold is similar to the ceramic rings coated by $2 \mu \mathrm{m}$-gold, a prototype of ceramic rings-loaded vacuum chamber with $0.5 \mu \mathrm{m}$-gold coating and rf shoulder will be manufactured and the impedance measurements will be performed.

\section{ACKNOWLEDGMENTS}

Thank the support from the National Natural Science Foundation of China (No. 11705253) and the support from Guangdong Innovative and Entrepreneurial Research Team Program (No. 2016ZT06G373).
[1] J. C. Yang, J. W. Xia, G. Q. Xiao et al., High intensity heavy ion accelerator facility in China, Nucl. Instrum. Methods Phys. Res., Sect. B 317, 263 (2013).

[2] S. Ruan, J. C. Yang, J. Q. Zhang et al., Design of extraction system in BRing at HIAF, Nucl. Instrum. Methods Phys. Res., Sect. A 892, 53 (2018).

[3] A.W. Chao, Physics of Collective Beam Instabilities in High Energy Accelerators (Wiley, New York, 1993).

[4] K. Y. Ng, Physics of Intensity Dependent Beam Instabilities (World Scientific, Singapore, 2006).

[5] Computer Simulation Technology. http://www.cst.com/.

[6] J. Liu, J. C. Yang, J. W. Xia et al., Transverse impedances and collective instabilities in a heavy ion accelerator, Phys. Rev. Accel. Beams 21, 064403 (2018).

[7] X. Q. Chen, J. C. Yang, J. W. Xia, G. D. Shen, S. Ruan, G. Wang, J. Liu, J. J. Zhang, and F. C. Cai, Study of eddy current effect in BRing at HIAF, Nucl. Instrum. Methods Phys. Res., Sect. A 920, 37 (2019).

[8] W. J. Xie, J. Meng, C. Luo, and Y. P. Wang, Baking-Power and thermal structure coupling of vacuum chamber of high intensity heavy ion accelerator: A simulation study, Chin. J. Vac. Sci. Technol. 37, 424 (2017).

[9] S.S. Kurennoy, Using a ceramic chamber in kicker magnets, in Proceedings of the 15th Particle Accelerator Conference, PAC-1993, Washington, DC, 1993 (IEEE, New York, 1993), p. 3420.

[10] R. L. Gluckstern, J. van Zeijts, and B. Zotter, Coupling impedance of beam pipes of general cross section, Report No. CERN SL/AP 92-25, 1992.

[11] K. Y. Ng and K. Bane, Explicit expressions of impedances and wake functions, Report No. FERMILAB-FN-0901APC, 2010.

[12] S. S. Kurennoy, Coupling impedance of pumping holes, Part. Accel. 39, 1 (1992).

[13] S. S. Kurennoy and G. Stupakov, A new method for calculating low frequency coupling impedance, Part. Accel. 45, 95 (1994).

[14] F. Caspers, E. Jensen, F. Ruggiero et al., RF screening by thin resistive layers, in Proceedings of the 18th Particle Accelerator Conference, New York, 1999 (IEEE, New York, 1999).

[15] H. A. Day, Measurements and simulations of impedance reduction techniques in particle accelerators, $\mathrm{Ph} . \mathrm{D}$. thesis, Manchester U, 2013.

[16] L. Walling et al., Transmission-line impedance measurements for an advanced hadron facility, Nucl. Instrum. Methods Phys. Res. 281, 433 (1989).

[17] T. Kroyer, F. Caspers, and E. Gaxiola, Longitudinal and transverse wire measurements for the evaluation of impedance reduction measures on the MKE extraction kickers, CERN Report No. AB-Note-2007-028.

[18] N. Baboi, R. M. Jones et al., Wire measurement of impedance of an x-band accelerator structure, in Proceedings of the 8th European Particle Accelerator Conference, Paris, 2002 (EPS-IGA and CERN, Geneva, 2002), p. 1440. 